\title{
Dualisation of the General Scalar Coset in Supergravity Theories
}

\author{
Nejat T. Yilmaz \\ Department of Physics \\ Middle East Technical University \\ 06531 Ankara,Turkey \\ ntyilmaz@metu.edu.tr
}

October 26, 2018

\begin{abstract}
The dualised formulation of the symmetric space sigma model is peformed for a general scalar coset $G / K$ where $G$ is a maximally noncompact group and $K$ it's maximal compact subgroup.By using the twisted self-duality condition the general form of the first-order equations are obtained.The results are applied to the example of $S L(2, \mathbb{R})$ $/ S O(2)$ scalar manifold of the IIB supergravity.
\end{abstract}

\section{Introduction}

In supergravity theories which possess scalar fields the global symmetries of the scalar sector are essential to have a deeper understanding of these theories. The scalar Lagrangians can be formulated as coset sigma models which are non-linear realisations. The dimension of the coset space is equal to the number of the scalars of the theory When we use the Kaluza-Klein dimensional reduction for $\mathrm{D}=11$ supergravity [1] and obtain the lower dimensional maximal supergravity theories the global (rigid) symmetry group (i.e.a real Lie group) of the Bosonic sector of the reduced Lagrangians are in split real form (maximally non-compact whose Cartan subalgebras can be chosen along the non-compact directions). This global symmetry is also the 
global symmetry of the scalars of the theory.The scalar coset can be constructed as $G / K$-valued fields where $G$ is the global symmetry group in split real form and $K$ it's maximal compact subgroup. The scalars transform nonlinearly in the linear representations of $G$ while the higher rank potentials always transform linearly.Although the global symmetry scheme of the supergravity theories are consequences of supersymmetry the study of the coset symmetries of the supergravity theories provides a better understanding of the underlying structure of these theories $[2,3]$.

The method of non-linear realisations $[4,5,6,7,8]$ is used in $[9,10]$ to formulate the gravity as a non-linear realisation in which the gravity and the gauge fields appear on equal footing. Recently this formulation is enlarged to have a detailed treatment of the maximal supergravity theories.

By introducing auxilary fields for a subset of the field content and by using the coset formulation the global symmetries of the scalar sectors of maximal supergravities are studied in [11].These symmetries which are realised on the Bosonic fields are also studied in a general formalism in [12]. However the complete coset realisation of the scalars and the gauge fields is introduced in [13] where the twisted self-duality structure of supergravities $[14,15]$ is generalised to regain the first-order equations of the theory from the Cartan forms of the dualised coset.The spacetime symmetries turn out to be internal symmetries after the Kaluza-Klein dimensional reduction in [11].It is shown that these symmetries become gauge symmetries after dualisation. The discussion about the symmetry groups of the Cartan forms (the doubled field strengths) of the coset formulation as well as the symmetry groups of the twisted self-duality equation (i.e.first-order equations) which are larger than the symmetry groups of the Cartan forms is given in [11] and [13] .In [13] for IIB theory [16] it is also shown that the the global symmetry of the twisted self-duality equation of the Cartan form of the coset realisation is the original global symmetry. The same result is proved to exist for the dualisation of the scalar sectors of maximal supergravities in all dimensions and it is conjectured that the global symmetry is preserved for the full dualisation of these theories.

In [17] the complete non-linear realisation of the $\mathrm{D}=11$ and IIA $[18,19,20]$ supergravity theories is performed for the entire Bosonic sector including the gravity which is missing in [13].The symmetries are also discussed in detail in [17].In [21] the Bosonic sector of IIB is derived as a non-linear realisation. The non-linear realisations of $\mathrm{D}=11$ supergravity and IIA supergravity lead to finite dimensional Lie algebras $G_{11}$ and $G_{I I A}$ respectively which are not Kac- 
Moody algebras. The local subgroups are also chosen to be the Lorentz group so that the general coset representative of the entire Bosonic sector can not be parametrised by a Borel subgroup of some larger group .These coset formulations are not like the ones for maximal supergravities which all give rise to Kac-Moody algebras and whose general coset representatives can be parametrised by a Borel subgroup of a larger group.In [21,22] it is discussed that the non-linear realisations of $\mathrm{D}=11$ and IIA supergravities can be enlarged to include a Kac-Moody algebra identified as $E_{11}$ which contains the Borel subalgebra of $E_{8}$.This can be done either by introducing a larger local subgroup than the Lorentz group or by describing the gravity by two fields which are duals of each other.Furhermore in [21] the IIB theory is formulated as a non-linear realisation of an infinite dimensional algebra $G_{I I B}$ which is the closure of the conformal algebra.It is also shown that the non-linear realisation can be enlarged to include the Kac-Moody algebra $E_{11}$ of IIA and $\mathrm{D}=11$ supergravities.

In this note we will give the dualisation of the general symmetric space coset sigma model whose global (rigid) symmetry group is in split real form. The scalar sectors of the maximal supergravity theories are in this category and in [11] and [13] the scalar sectors are formulated case by case with in the context of the dualisation of the Bosonic sectors of these theories. The formulation presented here assumes a formal construction based on general structure constants and it is performed for a general $G / K$ scalar manifold ( $G$ is a maximally non-compact group and $K$ is it's maximal compact subgroup). The formulation is based on the Borel gauge as proposed in $[11,13]$ where the maximal supergravities with $\mathrm{D} \leq 9$ are based on a coset representative in which the axions appear as various exponential factors.A transformation of the fields is needed to relate the formulation given here and the one in [11] and [13] for $\mathrm{D} \leq 9$. On the other hand for IIB and IIA we have the exact match of the form of the coset representative chosen (IIA does not contain axions at all).Among the three possible formulations of the symmetric space sigma model [11] we will follow the one which defines an internal metric that is used instead of the coset element. Although the general form of the coset Lagrangian is given in [13] the explicit general dualised formulation is not performed.This work completes the missing general formulation by presenting the dualisation of the general coset $G / K$ by means of the abstract structure constants.It also contains the first-order formulation of the equations of motion as a twisted self-duality condition for the symmetric space coset sigma model with the global symmetry group in split real form. 
We will start with the derivation of the second-order equations of motion of the $G / K$ scalar coset manifold in section two.In section three we will calculate the general structure constants of the dualised algebra in terms of the ones of the originally assumed Borel subalgebra by introducing the dual fields and the dual generators.In section four the doubled field strength $\mathcal{G}$ will be calculated and the first-order equations will be derived by using the twisted self-duality equation $* \mathcal{G}=\mathcal{S G}$ (which is an exact self-duality condition since $\mathcal{S}=1$ in our case).Finally we will give an application of the results we derive for the example of $S L(2, \mathbb{R}) / S O(2)$ scalar manifold in section five.

\section{The General Scalar Coset Sigma Model}

In this section we will derive the equations of motion for the scalar coset after we introduce the Lagrangian. The formulation is parallel with the one in [23]. The equations are second-order and the dualisation will enable us to obtain the first-order equations of motion.Let $G$ be a maximally non-compact (split) group and $K$ it's maximal compact subgroup.One can consider the $G$-valued scalar fields over the D-dimensional spacetime to form the scalar manifold first and then demand the local $K$ invariance. This will reduce the degrees of freedom and the physical scalar manifold elements will be the $G / K$ coset-valued fields. The coset representatives can be parametrised by the Borel subgroup elements. The Borel subgroup is generated by the Borel subalgebra whose generators are the Cartan generators $\left\{H_{i} \mid i=1, . ., l\right\}$ of the Cartan subalgebra and the positive root generators $\left\{E_{\alpha} \mid \alpha=1, . ., m\right\}$ of the Lie algebra of $G$.So the $G / K$-valued field $\nu(x)$ can be written as

$$
\begin{aligned}
\nu(x) & =\mathbf{g}_{H}(x) \mathbf{g}_{N}(x) \\
& =e^{\frac{1}{2} \phi^{i}(x) H_{i}} e^{\chi^{m}(x) E_{m}} .
\end{aligned}
$$

In this so-called Borel gauge the fields $\left\{\phi^{i}\right\}$ which couple to $\left\{H_{i}\right\}$ are called the dilatons and the fields $\left\{\chi^{m}\right\}$ which couple to the positive root generators $\left\{E_{m}\right\}$ are called the axions. Totally there are $n=l+m$ scalar fields which generate the $G / K$-valued fields $\nu(x)$ of the scalar manifold.

One can parametrise the positive root part of $\nu(x)$ in various ways.For example like in $[11,13]$ the positive root part in $(2.1)$ can be constructed as products of exponentials of individual positive root generators multiplied 
with the corresponding axions. The factors are ordered in a special way so that the axions chosen are the same fields which appear in the particular form of the Lagrangians used. The Campbell-Hausdorff formula can be used to relate the field content (dilatons and axions) of the different parametrisations of $\nu(x)$.Indeed each parametrisation corresponds to a transformation of the scalar fields but we should also bear in mind that the Borel gauge of the coset does not change as in all of the parametrisations mentioned above the $G / K$ coset is still parametrised by the Borel subgroup.

If we define the right,rigid action of the group $G$ over the $G / K$-valued fields as $\psi(x)=\nu(x) \mathbf{g}$ for $\mathbf{g} \in G$ we see that $\psi(x)$ is not in the Borel gauge .We should introduce another transformation which would map it into the range of the Borel gauge again. The Iwasawa decomposion [24] is the tool for this.It assures that any group element $\mathrm{g} \in G$ which is in the range of the exponential map can uniquely be expressed as

$$
\mathbf{g}=\mathbf{g}_{K} \mathbf{g}_{H} \mathbf{g}_{N}
$$

where $\mathbf{g}_{K} \in K$ while $\mathbf{g}_{H}$ and $\mathbf{g}_{N}$ are the group elements obtained by the exponentiation of the Cartan subalgebra and the positive root part of the Lie algebra of $G$ respectively. Thus if we use this result for $\psi(x)$ we have $\psi(x)=$ $\mathbf{g}_{K}(x) \nu^{\prime}(x)$ where $\nu^{\prime}(x)=\mathbf{g}_{K}^{-1}(x) \psi(x)$ is in the Borel gauge.Consequently for each rigid transformation $\mathbf{g} \in G$ there exists a local transformation $\mathbf{g}_{K}^{-1}(x) \in$ $K$ which enables the map

$$
\nu^{\prime}(x)=\mathbf{g}_{K}^{-1}(x) \nu(x) \mathbf{g}
$$

to be closed over the range of the Borel gauge. The general scalar coset Lagrangian is given as $[11,13,23]$

$$
\mathcal{L}=\frac{1}{4} \operatorname{tr}\left(* d \mathcal{M}^{-1} \wedge d \mathcal{M}\right) .
$$

Here the internal metric $\mathcal{M}$ is $\mathcal{M}=\nu^{\#} \nu$ where ()$^{\#}$ is the generalised transpose.In order to define it we first introduce $\tau: g \rightarrow g$ namely the Cartan involution which acts on $g$ the Lie algebra of $G$.It reverses the sign of the non-compact generators while leaving the compact generators unchanged.In terms of the basis we use

$$
\tau:\left(E_{\alpha}, E_{-\alpha}, H_{i}\right) \longrightarrow\left(-E_{-\alpha},-E_{\alpha},-H_{i}\right)
$$


where $\left\{E_{-\alpha}\right\}$ are the negative root generators.For any real group the compact generators can be written as $\left(E_{\alpha}-E_{-\alpha}\right)$ and the non-compact ones as $\left(E_{\alpha}+E_{-\alpha}\right)$.For a Lie algebra element $g^{\prime}$ the general transpose \# is defined as $\left(g^{\prime}\right)^{\#}=-\tau\left(g^{\prime}\right)$. As mentioned in [23] it is possible to find a higher dimensional representation of the Lie algebra in which \# coincides with the matrix transpose operator.For this reason one can define a conjugate \# map over the group $G$ as $\left(\exp g^{\prime}\right)^{\#}=\exp \left(g^{\prime \#}\right)$.If the subgroup of $G$ generated by the compact generators is an orthogonal group then in the fundamental representation the generators can be chosen such that $\mathbf{g}^{\#}=\mathbf{g}^{\mathbf{\top}}($ i.e. $S L(n, \mathbb{R}))$.If it is a unitary group then $\mathbf{g}^{\#}=\mathbf{g}^{\dagger}$. Therefore the generalized transpose of the coset representative is defined as follows

$$
\begin{aligned}
\nu^{\#} & =\tau\left(\nu^{-1}\right) \\
& =e^{\chi^{m} E_{-m}} e^{\frac{1}{2} \phi^{i} H_{i}} .
\end{aligned}
$$

The Iwasawa decomposition assures that the Lagrangian (2.4) is invariant under the rigid transformations of $G$ which we have defined before.As discussed in [13] the transformation acting on the general Noether currents generated by the rigid (non-coordinate dependent) right action of $G$ on the scalar coset manifold is linear. Since the number of currents exceeds the total number of scalars (dilatons and axions) of the theory these currents are not all independent. Therefore the Borel currents corresponding to the symmetries generated by the Borel subalgebra transform non-linearly under the action of $G$.This may be phrased as the non-linear realisation of the global symmetry group $G$ over the scalars.

By using $\nu^{-1} d \nu=-d \nu^{-1} \nu$ and the properties of the generalized transpose \# also the fact that the cyclic permutations are permissable under the trace the scalar coset Lagrangian can be expressed as

$$
\mathcal{L}=-\frac{1}{2} \operatorname{tr}\left(* d \nu \nu^{-1} \wedge\left(d \nu \nu^{-1}\right)^{\#}+* d \nu \nu^{-1} \wedge d \nu \nu^{-1}\right) .
$$

As mentioned in section one the scalar coset manifolds of the maximal supergravity theories can be given as $G / K, G$ being a maximally non-compact group and $K$ it's maximal compact subgroup.For $\mathrm{D}=11$ supergravity the global symmetry group $G$ is the trivial group,for IIB $G$ is $S L(2, \mathbb{R})$ and $K$ is $S O(2)$,for IIA $G$ is $S O(1,1) / \mathbb{Z}_{2}$ and $K$ is the trivial group. The $\mathrm{D}=9$ maximal supergravity has the scalar coset $G L(2, \mathbb{R}) / S O(2)$.Exceptional groups $E_{n}$ 
arise as the global symmetry groups for $D<9$ maximal supergravities. The details of the maximal compact subgroups of these theories can be found in $[24]$.

The 1-form $\mathcal{G}_{0}=d \nu \nu^{-1}$ is the pullback of a Lie algebra valued 1-form on $G$ through the map $\nu$.For this reason it can be expressed in the Borel subalgebra basis

$$
\begin{aligned}
\mathcal{G}_{0} & =d \nu \nu^{-1} \\
& =\left(d \mathbf{g}_{H} \mathbf{g}_{N}+\mathbf{g}_{H} d \mathbf{g}_{N}\right)\left(\mathbf{g}_{N}^{-1} \mathbf{g}_{H}^{-1}\right) \\
& =d \mathbf{g}_{H} \mathbf{g}_{H}^{-1}+\mathbf{g}_{H} d \mathbf{g}_{N} \mathbf{g}_{N}^{-1} \mathbf{g}_{H}^{-1} .
\end{aligned}
$$

Now in order to simplify the first term we will use the formula

$$
d e^{X} e^{-X}=d X+\frac{1}{2 !}[X, d X]+\frac{1}{3 !}[X,[X, d X]]+\ldots
$$

Therefore we have

$$
\begin{aligned}
d \mathbf{g}_{H} \mathbf{g}_{H}^{-1} & =d e^{\frac{1}{2} \phi^{i} H_{i}} e^{-\frac{1}{2} \phi^{i} H_{i}} \\
& =\frac{1}{2} d \phi^{i} H_{i}
\end{aligned}
$$

where we have used the fact that $\left[H_{i}, H_{j}\right]=0$.The other commutation relations of the Borel subalgebra are $\left[H_{i}, E_{\alpha}\right]=\alpha_{i} E_{\alpha}$ and $\left[E_{\alpha}, E_{\beta}\right]=$ $N_{\alpha, \beta} E_{\alpha+\beta}$. By using the above expansion for $d e^{X} e^{-X}$ we can also calculate 
$d \mathbf{g}_{N} \mathbf{g}_{N}^{-1}$

$$
\begin{aligned}
d \mathbf{g}_{N} \mathbf{g}_{N}^{-1} & =d e^{\chi^{m} E_{m}} e^{-\chi^{m} E_{m}} \\
& =d \chi^{m} E_{m}+\frac{1}{2 !}\left[\chi^{m} E_{m}, d \chi^{n} E_{n}\right]+\frac{1}{3 !}\left[\chi^{m} E_{m},\left[\chi^{l} E_{l}, d \chi^{n} E_{n}\right]\right]+\ldots \\
& =d \chi^{m} E_{m}+\frac{1}{2 !} \chi^{m} d \chi^{n} K_{m n}^{v} E_{v}+\frac{1}{3 !} \chi^{m} \chi^{l} d \chi^{n} K_{l n}^{v} K_{m v}^{u} E_{u}+\ldots \\
& =\overrightarrow{\mathbf{E}} \Omega \overrightarrow{d \chi}
\end{aligned}
$$

In the compact form on the right hand side of the last line $\overrightarrow{\mathbf{E}}$ is the row vector of the positive root generators; $\mathbf{E}_{\alpha}=E_{\alpha}$ and $\overrightarrow{d \chi}$ is the column vector of the 1 -forms $\left\{d \chi^{\alpha}\right\}$. The matrix $\Omega$ is a series which arises from the infinite sum given above

$$
\boldsymbol{\Omega}=\sum_{n=0}^{\infty} \frac{\omega^{n}}{(n+1) !}
$$

$\omega$ is an $m \times m$ matrix $m$ being the number of the positive roots and it is composed of the axions coupled to the structure constants $\omega_{\beta}^{\gamma}=\chi^{\alpha} K_{\alpha \beta}^{\gamma}$.In this definition we have introduced $\left[E_{\alpha}, E_{\beta}\right]=K_{\alpha \beta}^{\gamma} E_{\gamma}$.In other words $K_{\beta \beta}^{\alpha}=$ $0, K_{\beta \gamma}^{\alpha}=N_{\beta, \gamma}$ if in the root sense $\beta+\gamma=\alpha$ and $K_{\beta \gamma}^{\alpha}=0$ if $\beta+\gamma \neq \alpha$ again in the root sense. Now if we use the Campbell-Hausdorff formula

$$
e^{X} Y e^{-X}=Y+[X, Y]+\frac{1}{2 !}[X,[X, Y]]+\ldots
$$

we can explicitly write the second term in (2.8) as

$$
\begin{aligned}
\mathbf{g}_{H} d \mathbf{g}_{N} \mathbf{g}_{N}^{-1} \mathbf{g}_{H}^{-1} & =e^{\frac{1}{2} \phi^{i} H_{i}}(\overrightarrow{\mathbf{E}} \boldsymbol{\Omega} \overrightarrow{d \chi}) e^{-\frac{1}{2} \phi^{i} H_{i}} \\
& =\overrightarrow{\mathbf{E}^{\prime}} \boldsymbol{\Omega} \overrightarrow{d \chi}
\end{aligned}
$$


The primed row vector $\overrightarrow{\mathbf{E}}^{\prime}$ is defined as $\mathbf{E}_{\beta}^{\prime}=e^{\frac{1}{2} \beta_{i} \phi^{i}} E_{\beta}$. Finally we can now write $\mathcal{G}_{0}$ expanded in the Borel subalgebra generators

$$
\begin{aligned}
\mathcal{G}_{0} & =d \nu \nu^{-1} \\
& =\frac{1}{2} d \phi^{i} H_{i}+e^{\frac{1}{2} \alpha_{i} \phi^{i}} F^{\alpha} E_{\alpha}
\end{aligned}
$$

We have also introduced the vector $F^{\alpha}=\Omega_{\beta}^{\alpha} d \chi^{\beta}$. We will express the equations of motion in terms of $\overrightarrow{\mathbf{F}}$.If we use (2.15) in (2.7) we find that

$$
\mathcal{L}=-\frac{1}{2} \sum_{i=1}^{l} * d \phi^{i} \wedge d \phi^{i}-\frac{1}{2} \sum_{\alpha=1}^{m} e^{\alpha_{i} \phi^{i}} * F^{\alpha} \wedge F^{\alpha} .
$$

By following the outline of [23] one can derive the equations of motion of the general scalar coset Lagrangian. We should first observe that $d\left(d \nu \nu^{-1}\right)=$ $-d \nu \wedge d \nu^{-1}=d \nu \nu^{-1} \wedge d \nu \nu^{-1}$.If (2.15) is substituted into this equation one gets the Bianchi identity for $\overrightarrow{\mathbf{F}}$.

$$
d F^{\gamma}=\frac{1}{2} \sum_{\alpha+\beta=\gamma} N_{\alpha, \beta} F^{\alpha} \wedge F^{\beta} .
$$

If $\left\{F^{\gamma}\right\}$ are considered as independent fields and if we propose the Bianchi identity as a constraint equation the Lagrange multipliers ( $(D-2)$-forms) can be introduced and the additional Lagrangian corresponding to the Bianchi identity can be given as

$$
\mathcal{L}_{\text {Bianchi }}=\left(d F^{\alpha}-\frac{1}{2} \sum_{\alpha=\beta+\gamma} N_{\beta, \gamma} F^{\beta} \wedge F^{\gamma}\right) \wedge A_{(D-2), \alpha} .
$$

The new Lagrangian becomes $\mathcal{L}^{\prime}=\mathcal{L}+\mathcal{L}_{\text {Bianchi }}$. The variation with respect to $A_{(D-2), \alpha}$ for $\alpha=1, . ., m$ will give back the Bianchi identities.If we vary $\mathcal{L}^{\prime}$ with respect to $F^{\gamma}$ and then take the exterior derivative of the resulting field equation we achieve the second-order equations of motion for $F^{\gamma}$

$$
d\left(e^{\gamma_{i} \phi^{i}} * F^{\gamma}\right)=\sum_{\alpha-\beta=-\gamma} N_{\alpha,-\beta} F^{\alpha} \wedge e^{\beta_{i} \phi^{i}} * F^{\beta} .
$$


By varying (2.16) with respect to the dilatons $\left\{\phi^{i}\right\}$ (since $\mathcal{L}_{\text {Bianchi }}$ does not depend on $\left\{\phi^{i}\right\}$ ) we can also find the equations of motion for $\phi^{i}$

$$
d\left(* d \phi^{i}\right)=\frac{1}{2} \sum_{\alpha=1}^{m} \alpha_{i} e^{\frac{1}{2} \alpha_{i} \phi^{i}} F^{\alpha} \wedge e^{\frac{1}{2} \alpha_{i} \phi^{i}} * F^{\alpha} .
$$

The details of the formulation given above can be found in [23].As we will see in the next section in order to formulate the dualised theory we need to use a slightly different form of (2.19) namely

$$
\begin{aligned}
d\left(e^{\frac{1}{2} \gamma_{i} \phi^{i}} * F^{\gamma}\right) & =d\left(e^{-\frac{1}{2} \gamma_{i} \phi^{i}} e^{\gamma_{i} \phi^{i}} * F^{\gamma}\right) \\
& =-\frac{1}{2} \gamma_{j} e^{-\frac{1}{2} \gamma_{i} \phi^{i}} d \phi^{j} \wedge e^{\gamma_{i} \phi^{i}} * F^{\gamma} \\
& +\sum_{\alpha-\beta=-\gamma} e^{-\frac{1}{2} \gamma_{i} \phi^{i}} N_{\alpha,-\beta} F^{\alpha} \wedge e^{\beta_{i} \phi^{i}} * F^{\beta} \\
& =-\frac{1}{2} \gamma_{j} e^{\frac{1}{2} \gamma_{i} \phi^{i}} d \phi^{j} \wedge * F^{\gamma}+\sum_{\alpha-\beta=-\gamma} e^{\frac{1}{2} \alpha_{i} \phi^{i}} e^{\frac{1}{2} \beta_{i} \phi^{i}} N_{\alpha,-\beta} F^{\alpha} \wedge * F^{\beta} .
\end{aligned}
$$

The second-order equations (2.20) and (2.21) are the ones which we will refer to derive the commutation relations of the dualised generators when we construct the doubled field strength $\mathcal{G}$ which will give the correct first-order equations by satisfying a twisted self-duality condition.

\section{Dualisation}

If we double the number of the fields by introducing a $(D-2)$-form for each scalar field we can construct a doubled field strength $\mathcal{G}$ which is Lie superalgebra valued $[11,13]$.This algebra is generated by the Borel generators and the generators we introduce for each dual $(D-2)$-form whose commutation relations we will calculate.As we will see the choice of the appropriate structure constants will be based on the formulation which will give the correct equations of motion. The doubled field strength $\mathcal{G}$ is invariant under the Borel 
subgroup $G_{+}$of $G$ which corresponds to the rigid symmetries of the constant shifts of the dilatons and the axions and $\mathcal{G}$ is also invariant under the local gauge symmetries which are generated by the generators coupled to the dual $(D-2)$-forms as discussed in [13].For the doubled field content we propose the map $\nu^{\prime}(x)$ as

$$
\nu^{\prime}(x)=e^{\frac{1}{2} \phi^{i} H_{i}} e^{\chi^{m} E_{m}} e^{\widetilde{\chi}^{m} \widetilde{E}_{m}} e^{\frac{1}{2} \widetilde{\phi}^{i} \widetilde{H}_{i}} .
$$

The doubled field strength $\mathcal{G}$ is defined as $d \nu^{\prime} \nu^{\prime-1}$.In [11] and [13] the twisted self-duality condition is applied on $\mathcal{G}$ to regain the first-order equations of the maximal supergravity theories. The twisted self-duality condition which is imposed on $\mathcal{G}$ is $* \mathcal{G}=\mathcal{S G}$. Here $\mathcal{S}$ is a pseudo-involution of the proposed Lie superalgebra (which is generated by the original Borel generators and their duals).It's action on the scalar and dual generators is as follows

$$
\begin{array}{ll}
\mathcal{S} H_{i}=\widetilde{H}_{i} \quad, \quad \mathcal{S} E_{m}=\widetilde{E}_{m}, \\
\mathcal{S} \widetilde{H}_{i}=H_{i} \quad, \quad \mathcal{S} \widetilde{E}_{m}=E_{m} .
\end{array}
$$

In general $\mathcal{S}$ sends the Borel generators to their duals and the dual ones to their Borel counterparts with a sign factor which is $(-1)^{p(D-p)+s}$ where $p$ is the degree of the corresponding field strength which the generator is coupled to and $s$ is the signature of the spacetime metric.In fact the eigenvalues of $\mathcal{S}^{2}$ must be the same with the eigenvalues of $(* \circ *)$ operator acting on the field strength coupled to the dual generator which $\mathcal{S}^{2}$ acts on.For the present case the field strengths are $(D-1)$-forms so $p=D-1$ and the signature of the spacetime is assumed to be $s=D-1$. So the sign factor is $(-1)^{p(D-p)+s}=(-1)^{2(D-1)}=1$.

The Borel generators satisfy the commutation relations given in the previous section and as mentioned in [13] the general form of the remaining commutation relations of the newly constructed algebra are

$$
\begin{gathered}
{\left[E_{\alpha}, \widetilde{T}_{m}\right]=\widetilde{f}_{\alpha m}^{n} \widetilde{T}_{n} \quad, \quad\left[H_{i}, \widetilde{T}_{m}\right]=\widetilde{g}_{i m}^{n} \widetilde{T}_{n}} \\
{\left[\widetilde{T}_{m}, \widetilde{T}_{n}\right]=0}
\end{gathered}
$$


where $\widetilde{T}_{i}=\widetilde{H}_{i}$ for $i=1, \ldots, l$ and $\widetilde{T}_{\alpha+l}=\widetilde{E}_{\alpha}$ for $\alpha=1, \ldots, m$.In the next section we will calculate the doubled field strength $\mathcal{G}=d \nu^{\prime} \nu^{\prime-1}$ explicitly but the following formulation will make use of an alternative method introduced and used in [13]. We will define a the field strength $\mathcal{G}^{\prime}$ as $\mathcal{G}^{\prime}=\mathcal{G}_{0}+\mathcal{S} * \mathcal{G}_{0}$. By using (2.15)

$$
\mathcal{G}^{\prime}=d \nu \nu^{-1}+\frac{1}{2} * d \phi^{i} \widetilde{H}_{i}+e^{\frac{1}{2} \alpha_{i} \phi^{i}} * F^{\alpha} \widetilde{E}_{\alpha}
$$

From it's definition $\mathcal{G}^{\prime}$ trivially satisfies the twisted self-duality condition $* \mathcal{G}^{\prime}=\mathcal{S G}^{\prime}$. Since $d\left(d \nu \nu^{-1}\right)=d \nu \nu^{-1} \wedge d \nu \nu^{-1} \mathcal{G}_{0}$ satisfies the Cartan-Maurer equation $d \mathcal{G}_{0}-\mathcal{G}_{0} \wedge \mathcal{G}_{0}=0$. As a matter of fact $\mathcal{G}^{\prime}$ is nothing but $\mathcal{G}$ in which the self-duality condition $* \mathcal{G}=\mathcal{S G}$ is used for this reason $\mathcal{G}^{\prime}$ also satisfies the Cartan-Maurer equation

$$
d \mathcal{G}^{\prime}-\mathcal{G}^{\prime} \wedge \mathcal{G}^{\prime}=0 .
$$

This equation gives the original second-order equations of the coset Lagrangian. Therefore one way of obtaining the structure constants in (3.3) is to calculate (3.5) by substituting (3.4) and to compare the result with the second-order equations (2.20) and (2.21).

We will use this fact to read the structure constants in (3.3) in terms of the information of the Borel subalgebra of $G$. We should bear in mind that the generators $H_{i}$ and $E_{\alpha}$ are of even degree and the ones $\widetilde{H}_{i}$ and $\widetilde{E}_{\alpha}$ are of even or odd degree whether their corresponding potentials $(D-2)$-forms are even or odd rank depending on the spacetime dimension.In the exterior algebra of the Lie superalgebra valued forms even or odd generators behave like even or odd degree forms when commuting with the differential forms.Also if $T$ is an odd generator $d(T A)=-T d A$ and if it is even $d(T A)=T d A$. Now by using (3.4) if we calculate $\mathrm{d} \mathcal{G}^{\prime}-\mathcal{G}^{\prime} \wedge \mathcal{G}^{\prime}$ and equate it to zero we find that

$$
\begin{aligned}
d \mathcal{G}^{\prime}-\mathcal{G}^{\prime} \wedge \mathcal{G}^{\prime} & =\frac{1}{2} d\left(* d \phi^{i}\right) \widetilde{H}_{i}+d\left(e^{\frac{1}{2} \alpha_{i} \phi^{i}} * F^{\alpha}\right) \widetilde{E}_{\alpha}-\frac{1}{4} d \phi^{j} \wedge * d \phi^{i}\left[H_{j}, \widetilde{H}_{i}\right] \\
& -\frac{1}{2} d \phi^{j} \wedge e^{\frac{1}{2} \alpha_{i} \phi^{i}} * F^{\alpha}\left[H_{j}, \widetilde{E}_{\alpha}\right]-\frac{1}{2} e^{\frac{1}{2} \alpha_{i} \phi^{i}} F^{\alpha} \wedge * d \phi^{j}\left[E_{\alpha}, \widetilde{H}_{j}\right] \\
& -e^{\frac{1}{2} \alpha_{i} \phi^{i}} e^{\frac{1}{2} \beta_{i} \phi^{i}} F^{\alpha} \wedge * F^{\beta}\left[E_{\alpha}, \widetilde{E}_{\beta}\right]
\end{aligned}
$$


We have to equate the coefficients of the linearly independent algebra generators to zero and the resulting equations must be the same with the second-order equations of motion (2.20) and (2.21).Direct comparison gives the commutation relations as

$$
\begin{gathered}
{\left[H_{j}, \widetilde{H}_{i}\right]=0 \quad, \quad\left[E_{\alpha}, \widetilde{H}_{j}\right]=0,} \\
{\left[H_{j}, \widetilde{E}_{\alpha}\right]=-\alpha_{j} \widetilde{E}_{\alpha} \quad, \quad\left[E_{\alpha}, \widetilde{E}_{\alpha}\right]=\frac{1}{4} \sum_{i=1}^{l} \alpha_{i} \widetilde{H}_{i},} \\
{\left[E_{\alpha}, \widetilde{E}_{\beta}\right]=N_{\alpha,-\beta} \widetilde{E}_{\gamma}, \quad \alpha-\beta=-\gamma, \alpha \neq \beta .}
\end{gathered}
$$

The conditions in the last line must be understood in the root sense.We can now express the structure constants $\widetilde{f}_{\alpha m}^{n}$ and $\widetilde{g}_{i m}^{n}$ by using the results we have found

$$
\begin{gathered}
\widetilde{f}_{\alpha m}^{n}=0, \quad m \leq l, \quad \tilde{f}_{\alpha, \alpha+l}^{i}=\frac{1}{4} \alpha_{i}, \quad i \leq l \\
\widetilde{f}_{\alpha, \alpha+l}^{i}=0, \quad i>l \quad, \quad \widetilde{f}_{\alpha, \beta+l}^{i}=0, \quad i \leq l, \alpha \neq \beta \\
\widetilde{f}_{\alpha, \beta+l}^{\gamma+l}=N_{\alpha,-\beta}, \quad \alpha-\beta=-\gamma \\
\widetilde{f}_{\alpha, \beta+l}^{\gamma+l}=0, \quad \alpha-\beta \neq-\gamma, \alpha \neq \beta .
\end{gathered}
$$

The conditions on the indices $\alpha$ and $\beta$ are in the root sense.Also we have

$$
\begin{gathered}
\widetilde{g}_{i m}^{n}=0, \quad m \leq l \quad, \quad \widetilde{g}_{i m}^{n}=0, \quad m>l, m \neq n \\
\widetilde{g}_{i \alpha}^{\alpha}=-\alpha_{i}, \quad \alpha>l .
\end{gathered}
$$




\section{The First-Order Equations of Motion}

Now that we have obtained the complete commutation relations of the dualised algebra we can explicitly calculate the doubled field strength in terms of the structure constants and then use the twisted self-duality equation $* \mathcal{G}=\mathcal{S G}$ to reach the first-order equations of motion. We should make the remark that although the symmetry group of $\mathcal{G}$ is composed of the Borel subgroup $G_{+}$and the local gauge symmetries of the dual generators the symmetry group which leaves the first-order equations $* \mathcal{G}=\mathcal{S G}$ is larger than that. The reason for this is that there could be symmetry transformations which may change $\mathcal{G}$ but still leave the twisted self-duality condition invariant.By using (3.1) we have

$$
\begin{aligned}
\mathcal{G}=d \nu^{\prime} \nu^{\prime-1} & =\mathcal{G}_{0}+e^{\frac{1}{2} \phi^{i} H_{i}} e^{\chi^{m} E_{m}} d e^{\widetilde{\chi}^{m} \widetilde{E}_{m}} e^{-\widetilde{\chi}^{m} \widetilde{E}_{m}} e^{-\chi^{m} E_{m}} e^{-\frac{1}{2} \phi^{i} H_{i}} \\
& +e^{\frac{1}{2} \phi^{i} H_{i}} e^{\chi^{m} E_{m}} e^{\widetilde{\chi}^{m} \widetilde{E}_{m}} d e^{\frac{1}{2} \widetilde{\phi}^{i} \widetilde{H}_{i}} e^{-\frac{1}{2} \widetilde{\phi}^{i} \widetilde{H}_{i}} e^{-\widetilde{\chi}^{m} \widetilde{E}_{m}} e^{-\chi^{m} E_{m}} e^{-\frac{1}{2} \phi^{i} H_{i}}
\end{aligned}
$$

Now by using the equation $(2.9)$ and the fact that $\left[\widetilde{E}_{m}, \widetilde{E}_{n}\right]=\left[\widetilde{H}_{i}, \widetilde{H}_{j}\right]=$ $\left[\widetilde{E}_{m}, \widetilde{H}_{i}\right]=0$ we obtain

$$
\begin{aligned}
& d e^{\widetilde{\chi}^{m} \widetilde{E}_{m}} e^{-\widetilde{\chi}^{m} \widetilde{E}_{m}}=d \widetilde{\chi}^{m} \widetilde{E}_{m} \\
& e^{\widetilde{\chi}^{m} \widetilde{E}_{m}} d e^{\frac{1}{2} \widetilde{\phi}^{i} \widetilde{H}_{i}} e^{-\frac{1}{2} \widetilde{\phi}^{i} \widetilde{H}_{i}} e^{-\widetilde{\chi}^{m} \widetilde{E}_{m}}=\frac{1}{2} d \widetilde{\phi}^{i} \widetilde{H}_{i} .
\end{aligned}
$$

So the field strength becomes

$$
\mathcal{G}=\mathcal{G}_{0}+e^{\frac{1}{2} \phi^{i} H_{i}} e^{\chi^{m} E_{m}} A^{n} \widetilde{T}_{n} e^{-\chi^{m} E_{m}} e^{-\frac{1}{2} \phi^{i} H_{i}}
$$

where we have defined the vector $\overrightarrow{\mathbf{A}}$ as $A^{i}=\frac{1}{2} d \widetilde{\phi}^{i}$ for $i=1, \ldots, l$ and $A^{i+\alpha}=d \widetilde{\chi}^{\alpha}$ for $\alpha=1, \ldots, m$.If we use the Campbell-Hausdorff formula (2.13) 
twice we can calculate the second term in (4.3)

$$
\begin{aligned}
e^{\chi^{m} E_{m}} A^{n} \widetilde{T}_{n} e^{-\chi^{m} E_{m}}= & A^{n} \widetilde{T}_{n}+\left[\chi^{m} E_{m}, A^{n} \widetilde{T}_{n}\right]+\frac{1}{2 !}\left[\chi^{m} E_{m},\left[\chi^{l} E_{l}, A^{n} \widetilde{T}_{n}\right]\right]+\ldots \\
= & A^{n} \widetilde{T}_{n}+\chi^{m} A^{n} \widetilde{f}_{m n}^{k} \widetilde{T}_{k}+\frac{1}{2 !} \chi^{m} \chi^{l} A^{n} \widetilde{f}_{m k}^{v} \widetilde{f}_{l n}^{k} \widetilde{T}_{v}+\ldots \\
& \overrightarrow{\widetilde{T}} e^{\boldsymbol{\Lambda}} \overrightarrow{\mathbf{A}} .
\end{aligned}
$$

We have defined the row vector $\overrightarrow{\widetilde{\mathbf{T}}}$ in section three and the matrix $\boldsymbol{\Lambda}$ is defined as $\Lambda_{n}^{k}=\chi^{m} \widetilde{f}_{m n}^{k}$. Now applying the Campbell-Hausdorff formula (2.13) once more

$$
\begin{aligned}
e^{\frac{1}{2} \phi^{i} H_{i}\left(\overrightarrow{\mathbf{T}} e^{\boldsymbol{\Lambda}} \overrightarrow{\mathbf{A}}\right) e^{-\frac{1}{2} \phi^{i} H_{i}}} & =\overrightarrow{\widetilde{\mathbf{T}}} e^{\boldsymbol{\Lambda}} \overrightarrow{\mathbf{A}}+\left[\frac{1}{2} \phi^{i} H_{i},\left(\overrightarrow{\widetilde{\mathbf{T}}} e^{\boldsymbol{\Lambda}} \overrightarrow{\mathbf{A}}\right)\right] \\
& +\frac{1}{2 !}\left[\frac{1}{2} \phi^{i} H_{i},\left[\frac{1}{2} \phi^{j} H_{j},\left(\overrightarrow{\widetilde{\mathbf{T}}} e^{\boldsymbol{\Lambda}} \overrightarrow{\mathbf{A}}\right)\right]\right]+\ldots \\
& =\overrightarrow{\widetilde{\mathbf{T}}} e^{\boldsymbol{\Lambda}} \overrightarrow{\mathbf{A}}+\frac{1}{2} \phi^{i}\left(e^{\boldsymbol{\Lambda}}\right)_{l}^{k} A^{l} \widetilde{g}_{i k}^{v} \widetilde{T}_{v} \\
& +\frac{1}{2 !} \frac{1}{4} \phi^{i} \phi^{j}\left(e^{\boldsymbol{\Lambda}}\right)_{l}^{k} A^{l} \widetilde{g}_{i s}^{u} \widetilde{g}_{j k}^{s} \widetilde{T}_{u}+\ldots \\
& =\overrightarrow{\widetilde{\mathbf{T}}} e^{\boldsymbol{\Gamma}} e^{\boldsymbol{\Lambda}} \overrightarrow{\mathbf{A}}
\end{aligned}
$$

where we have defined the matrix $\Gamma$ as $\Gamma_{n}^{k}=\frac{1}{2} \phi^{i} \widetilde{g}_{i n}^{k}$. We can now write 
the doubled field strength $\mathcal{G}$

$$
\begin{aligned}
\mathcal{G} & =\mathcal{G}_{0}+\overrightarrow{\widetilde{\mathbf{T}}} e^{\boldsymbol{\Gamma}} e^{\boldsymbol{\Lambda}} \overrightarrow{\mathbf{A}} \\
& =\frac{1}{2} d \phi^{i} H_{i}+\overrightarrow{\mathbf{E}^{\prime}} \boldsymbol{\Omega} \overrightarrow{d \chi}+\overrightarrow{\widetilde{\mathbf{T}}} e^{\boldsymbol{\Gamma}} e^{\boldsymbol{\Lambda}} \overrightarrow{\mathbf{A}}
\end{aligned}
$$

As we have expressed $\mathcal{G}$ in the basis $\left\{H_{i}, E_{\alpha}, \widetilde{H}_{i}, \widetilde{E}_{\alpha}\right\}$ finally we can find the first order equations of the scalar Lagrangian (2.7) by using the twisted self- duality equation $* \mathcal{G}=\mathcal{S G}$.If we substitute (4.6) into this equation we can read the first-order equations for the dilatons and the axions in terms of the dual $(D-1)$-form field strengths. So the first-order equations are obtained by equating the coefficients of the same generators on opposite sides of the equation $* \mathcal{G}=\mathcal{S G}$ and in compact form they can be written as

$$
* \vec{\Psi}=e^{\boldsymbol{\Gamma}} e^{\boldsymbol{\Lambda}} \overrightarrow{\mathrm{A}}
$$

In this vector equation the column vector $\vec{\Psi}$ is defined as $\Psi^{i}=\frac{1}{2} d \phi^{i}$ for $i=1, \ldots, l$ and $\Psi^{\alpha+l}=e^{\frac{1}{2} \alpha_{i} \phi^{i}} \Omega_{l}^{\alpha} d \chi^{l}$ for $\alpha=1, \ldots, m$ where $\boldsymbol{\Omega}$ is the $m \times m$ matrix defined in the second section. This concludes our formulation next we will give an application of the results we have obtained for the $S L(2, \mathbb{R})$ coset.

\section{The $S L(2, \mathbb{R}) / S O(2)$ Scalar Coset}

The $S L(2, \mathbb{R}) / S O(2)$ scalar coset Lagrangians arise when the Kaluza-Klein reduction is applied over Tori.For example when the pure gravity in $\mathrm{D}+2 \mathrm{di}$ mensions is reduced to $\mathrm{D}$ dimensions over the 2 -Torus $\mathrm{T}^{2}$ the global symmetry of the reduced scalar Lagrangian becomes $\mathbb{R} \times S L(2, \mathbb{R})$. The Scalar coset manifold of IIB supergravity also appears as $S L(2, \mathbb{R}) / S O(2)$. The dualisation of the $S L(2, \mathbb{R}) / S O(2)$ coset is studied in $[11,13]$. We will derive the same results by using the general formulation we have developed.The Lie algebra of $S L(2, \mathbb{R})$ namely $s l(2, \mathbb{R})$ is isomorphic to $s u(2)$ and it has three generators $\left\{H, E_{+}, E_{-}\right\}$so it's Cartan subalgebras are one dimensional and there is one positive root and one negative root.Therefore the Borel subalgebra is generated by $\left\{H, E_{+}\right\}$and they satisfy the commutation relation $\left[H, E_{+}\right]=2 E_{+}$ 
denoting $\alpha=2$.For $\operatorname{sl}(2, \mathbb{R})$ one can chose the following representation

$$
H=\left(\begin{array}{cc}
1 & 0 \\
0 & -1
\end{array}\right) \quad E_{+}=\left(\begin{array}{ll}
0 & 1 \\
0 & 0
\end{array}\right) \quad E_{-}=\left(\begin{array}{ll}
0 & 0 \\
1 & 0
\end{array}\right) .
$$

So that we can calculate $\nu$ as

$$
\nu=e^{\frac{1}{2} \phi H} e^{\chi E_{+}}=\left(\begin{array}{cc}
e^{\frac{1}{2} \phi} & \chi e^{\frac{1}{2} \phi} \\
0 & e^{-\frac{1}{2} \phi}
\end{array}\right) .
$$

Also since for $\operatorname{sl}(2, \mathbb{R})$ the generalized transpose is simply the matrix transpose

$$
\mathcal{M}=\nu^{\top} \nu=\left(\begin{array}{cc}
e^{\phi} & \chi e^{\phi} \\
\chi e^{\phi} & e^{-\phi}+\chi^{2} e^{\phi}
\end{array}\right) .
$$

Now from (2.4) we have the Lagrangian

$$
\mathcal{L}=-\frac{1}{2} * d \phi \wedge d \phi-\frac{1}{2} e^{2 \phi} * d \chi \wedge d \chi
$$

We only have one positive root so $K_{\alpha \beta}^{\gamma}=0, \omega=0$ and from $(2.12) \Omega=1$.If we use these results in (2.15) we have

$$
\begin{aligned}
\mathcal{G}_{0} & =d \nu \nu^{-1} \\
& =\frac{1}{2} d \phi H+e^{\phi} d \chi E_{+} .
\end{aligned}
$$

This could directly be calculated from (5.2). Since $\alpha=2, F=d \chi$ and $N_{1,1}=0$ from (2.19) and (2.20) we have the second-order equations of motion

$$
\begin{aligned}
d(* d \phi) & =e^{2 \phi} d \chi \wedge * d \chi, \\
d\left(e^{2 \phi} * d \chi\right) & =0 .
\end{aligned}
$$

By using (3.7) the commutation relations for the dual generators can be found as 


$$
\begin{gathered}
{[H, \widetilde{H}]=\left[E_{+}, \widetilde{H}\right]=0,} \\
{\left[H, \widetilde{E}_{+}\right]=-2 \widetilde{E}_{+} \quad, \quad\left[E_{+}, \widetilde{E}_{+}\right]=\frac{1}{2} \widetilde{H} .}
\end{gathered}
$$

We can now calculate the first-order equations from (4.7). We should first observe that if we use the commutation relations (5.7) in (3.8) and (3.9) or directly in (3.3) we find that

$$
\begin{array}{cc}
\widetilde{f}_{11}^{1}=\widetilde{f}_{11}^{2}=\widetilde{f}_{12}^{2}=0 \quad, \quad \widetilde{f}_{12}^{1}=\frac{1}{2}, \\
\widetilde{g}_{11}^{1}=\widetilde{g}_{11}^{2}=\widetilde{g}_{12}^{1}=0 \quad, \quad \widetilde{g}_{12}^{2}=-2 .
\end{array}
$$

In order to calculate the first-order equations we will first calculate the exponentials in (4.7).Since $\boldsymbol{\Gamma}_{n}^{k}=\frac{1}{2} \phi^{i} \widetilde{g}_{i n}^{k}$ and $\boldsymbol{\Lambda}_{n}^{k}=\chi^{m} \widetilde{f}_{m n}^{k}$ we have the $\boldsymbol{\Gamma}$ and $\Lambda$ matrices as

$$
\boldsymbol{\Gamma}=\left(\begin{array}{cc}
0 & 0 \\
0 & -\phi
\end{array}\right) \quad, \quad \boldsymbol{\Lambda}=\left(\begin{array}{cc}
0 & \frac{1}{2} \chi \\
0 & 0
\end{array}\right) .
$$

When we exponentiate these matrices and multiply the results we get

$$
\begin{gathered}
e^{\boldsymbol{\Gamma}}=\left(\begin{array}{cc}
1 & 0 \\
0 & e^{-\phi}
\end{array}\right) \quad, \quad e^{\boldsymbol{\Lambda}}=\left(\begin{array}{cc}
1 & \frac{1}{2} \chi \\
0 & 1
\end{array}\right), \\
e^{\boldsymbol{\Gamma}} e^{\boldsymbol{\Lambda}}=\left(\begin{array}{cc}
1 & \frac{1}{2} \chi \\
0 & e^{-\phi}
\end{array}\right) .
\end{gathered}
$$

The vectors in (4.7) are calculated from their definitions given in section three and section four and by using (5.8)

$$
\vec{\Psi}=\left(\begin{array}{c}
\frac{1}{2} d \phi \\
e^{\phi} d \chi
\end{array}\right) \quad, \quad \overrightarrow{\mathbf{A}}=\left(\begin{array}{c}
\frac{1}{2} \mathrm{~d} \widetilde{\phi} \\
\mathrm{d} \widetilde{\chi}
\end{array}\right) .
$$


When we substitute these results in the equation (4.7)

$$
\left(\begin{array}{c}
\frac{1}{2} * d \phi \\
e^{\phi} * d \chi
\end{array}\right)=\left(\begin{array}{cc}
1 & \frac{1}{2} \chi \\
0 & e^{-\phi}
\end{array}\right)\left(\begin{array}{c}
\frac{1}{2} d \widetilde{\phi} \\
d \widetilde{\chi}
\end{array}\right)
$$

from which we read the first-order equations of motion for the $S L(2, \mathbb{R})$ $/ S O(2)$ coset as

$$
\begin{aligned}
* d \phi & =d \widetilde{\phi}+\chi d \widetilde{\chi}, \\
e^{\phi} * d \chi & =e^{-\phi} d \widetilde{\chi} .
\end{aligned}
$$

These first-order equations are the same equations with the ones in [13] which are obtained by directly integrating the second-order equations (5.6).

\section{Conclusion}

We have derived the second-order equations of motion for a general scalar coset sigma model.By following the framework of [13] the general form of the structure constants and the first-order equations are derived for the doubled field strength formalism. We have shown that these general formulas lead to the correct results for the case of $S L(2, \mathbb{R}) / S O(2)$.

We have started from any given coset $G / K$ and derived the dualised formulation as well as the first-order equations in terms of the given knowledge of the original global symmetry group of the scalar Lagrangian. The results achieved in this work are applicable to the scalar sectors of the maximal supergravities but as they are derived for a general symmetric space sigma model with the abstract notion of the structure constants they would dualise and construct the first-order formulation of any other theory which assumes the same form.Especially the non-linear realisations of the supergravity theories in various dimensions in which there is matter coupling would include the scalar formalism introduced here. The dualisation of the scalar sector is a primary task and a good starting point for the non-linear realisation of any supergravity theory since it contains the knowledge of the global symmetry group of the Bosonic sector of the theory.This work in the most general sense relates the structure of the original scalar coset and the symmetry group of the doubled field strength from which the first-order equations are derived. 
In spite of the fact that we have given the formulation of the coset sigma models for the maximally non-compact global symmetry group a similar formulation can be performed for the general case of non-compact groups but in that case the role of the Borel subgroup must be replaced by the solvable subgroup.The formulation of the non-linear sigma models and in general the principle sigma models would follow the same pattern presented here.

\section{Acknowledgements}

This work has been supported by TUBITAK (The Scientific and Technical Research Council of Turkey).I would like to thank Prof Peter West and Prof Tekin Dereli for discussions and useful remarks.

\section{References}

[1] E.Cremmer,B.Julia and J.Scherk,'Supergravity theory in eleven dimensions', Phys. Lett. B76 (1978) 409.

[2] E.Cremmer and B.Julia,'The N=8 supergravity theory. The Lagrangian', Phys. Lett. B80 (1978) 48.

[3] E.Cremmer and B.Julia,'The SO(8) supergravity', Nucl. Phys. B156 (1979) 141.

[4] S.Coleman,J.Wess and B.Zumino,'Structure of phenomenological Lagrangians.1.', Phys. Rev. 177 (1969) 2239.

[5] K.Callan,S.Coleman,J.Wess and B.Zumino,' Structure of phenomenological Lagrangians. 2',Phys. Rev. 177 (1969) 2247.

[6] D.V.Volkov,Sov. J. Part. Nucl. 4 (1973) 3.

[7] D.V.Volkov and V.P.Akulov,'Possible universal neutrino interaction' JETP Letters 16 (1972) 438.

[8] D.V.Volkov and V.P.Akulov,'Is the neutrino a Goldstone particle?', Phys. Lett. B46 (1973) 109. 
[9] V.Ogievetsky,'Infinite-dimensional algebra of general covariance group as the closure of the finite dimensional algebras of conformal and linear groups' Nuovo. Cimento 8 (1973) 988.

[10] A.Borisov and V.Ogievetsky,'Theory of dynamical affine and conformal symmetries as the theory of gravitational field' Teor. Mat. Phys. 21 (1974) 329.

[11] E.Cremmer,B.Julia,H.Lū and C.N.Pope, 'Dualisation of dualities' ,Nucl. Phys. B523 (1998) 73, hep-th/9710119.

[12] H.Lū and C.N.Pope, 'p-brane solitons in maximal supergravities' , Nucl. Phys. B465 (1996) 127, hep-th/9512012

[13] E.Cremmer,B.Julia,H.Lū and C.N.Pope,'Dualisation of dualities II: Twisted self-duality of doubled fields and superdualities' ,Nucl. Phys. B535 (1998) 242, hep-th/9806106.

[14] B.Julia and H.Nicolai,'Conformal internal symmetry of 2d sigmamodels coupled to gravity and a dilaton',Nucl. Phys. B482 (1996) 431, hep-th/9608082.

[15] D.Bernard and B.Julia,'Twisted self-duality of dimensionally reduced gravity and vertex operators',Nucl. Phys. B547 (1999) 427, hep-th/9712254.

[16] J.Shwarz and P.West,'Symmetries and transformations of chiral $N=2$ $D=10$ supergravity', Phys. Lett. 126B (1983) 301.

[17] P.West, 'Hidden superconformal symmetry in M theory', JHEP 08 (2000) 007, hep-th/0005270.

[18] C.Campbell and P.West, ' $N=2, D=10$ non-chiral supergravity and it's spontaneous compactification', Nucl. Phys. B243 (1984) 112.

[19] M.Hug and M.Namazie,'Kaluza-Klein supergravity in ten dimensions', Class. Q. Grav. 2 (1985).

[20] F.Giani and M.Pernici, 'N=2 supergravity in ten dimensions', Phys. Lett. 126B (1983) 301. 
[21] I.Schnakenburg and P.West, 'Kac-Moody symmetries of IIB supergravity' ,Phys. Lett. B517 (2001) 421, hep-th/0107181.

[22] P.West, 'E(11) and $M$ theory',Class.Quant.Grav. 18 (2001) 4443., hep-th/0104081.

[23] A.Keurentjes,'The group theory of oxidation', hep-th/0210178.

[24] Y.Tanii,'Introduction to supergravities in diverse dimensions', hep-th/9802138. 